\title{
VELOCITIES OF ESCAPERS FROM UNSTABLE HIERARCHICAL TRIPLE STARS
}

\author{
L.KISELEVA ${ }^{1}$,J.ANOSOVA ${ }^{2}$, J.COLIN ${ }^{3}$ \\ ${ }^{1}$ Institute of Astronomy, Madingley Rd., \\ Cambridge CB3 OHA, UK \\ ${ }^{2}$ National Astronomical Observatory, Tokyo 181, Japan \\ ${ }^{3}$ Observatoire de Bordeaux, Floirac, France
}

We consider unstable hierarchical triple stars which are disrupted by the straightforward ejection and escape of the distant companion. The main aim of the present work is the study of velocities of escaping stars from these unstable systems, and the dependence of these velocities on the masses of the stars and on the distances of the escapers from the centers of mass of the remaining binary and of the triple system as a whole. Velocities of escapers are estimated for actual star systems. It is shown that in the triple star $\lambda$ Tau, when it becomes unstable as a result of mass transfer in its close semi-detached binary, the velocity of the escaping distant companion with mass $M \approx 0.7 M_{\odot}$ can be about $100 \mathrm{~km} / \mathrm{s}$. A possible application of these results to the problem of anomalous high-velocity stars in the Galaxy is discussed. 